\title{
Hot Topic
}

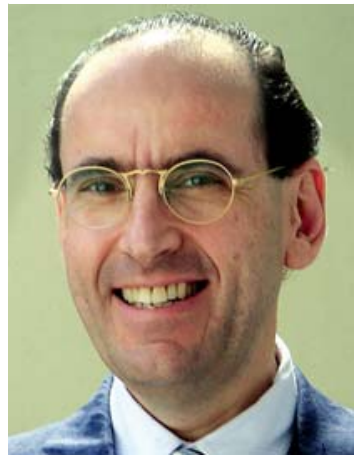

Dr. P. Kardos
Diese neue Rubrik in der Zeitschrift Pneumologie hat sich zum Ziel gesetzt, die deutschsprachige Leserschaft über neueste Meilensteine des Faches (oder solche, die zum Zeitpunkt der Publikation dafür gehalten werden) zeitnah zu informieren. Wir werden Artikel auswählen, die zwar unser Fach betreffen, aber so bedeutsam sind, dass sie nicht in pneumologischen Fachzeitschriften, sondern in renommierten medizinischen Zeitschriften wie das New England Journal of Medicine, The Lancet, Annals of Internal Medicine, British Medical Journal oder Nature Medicine publiziert werden. Sie sind nicht nur für den Spezialisten, sondern für die gesamte Ärzteschaft von brennendem Interesse.

„Hot“ und „brennend“ dürfen dabei wortwörtlich genommen werden: Wir bemühen uns, das „Hot Topic" in der jeweils nachfolgenden Ausgabe der Pneumologie zu publizieren. Wir haben den Ehrgeiz - falls eine Publikation bereits online vorliegt -, das „Hot Topic“ gar etwa zur gleichen Zeit mit der gedruckten Originalpublikation erscheinen zu lassen.

Wir hoffen, mit diesem Angebot eine Lücke zu füllen. Es ist schier unmöglich, alle bedeutsamen Publikationen aus pneumologischen Fachzeitschriften vollständig und zeitnah zu kommentieren. Wir versuchen zumindest, über Entwicklungen mit potenziell epochaler Bedeutung zeitnah $\mathrm{zu}$ berichten. Im Informationszeitalter sorgen eine große Anzahl elektronischer und Printmedien für Laien dafür, dass unsere Patienten sehr schnell über „Hot Topics“ mehr oder weniger zutreffende, manchmal bruchstückhafte Informationen erlangen und diese mit ihrem Arzt diskutieren möchten. Nichts ist unangenehmer in der Sprechstunde als ein Informationsvorsprung des zu beratenden Patienten!

Meilensteinartikel pflegen nicht nach einem gleichmäßigen Zeitplan zu erscheinen. Deshalb bitten wir um Verständnis, wenn diese Rubrik ebenfalls in unregelmäßigen Abständen ad hoc erscheinen wird. Ihr Herausgeberteam wird sich aber bemühen, solche Erscheinungen nicht zu übersehen. Gerne nehmen wir hierzu auch Vorschläge unserer Leserschaft entgegen, zu richten an die Herausgeber Prof. Tom Schaberg (schaberg@diako-online.de) oder Prof. Santiago Ewig (ewig@augusta-bochum.de).

Was könnte besser geeignet sein für unser erstes „Hot Topic“, wenn nicht der Ende Oktober 2011 im New England Journal of Medicine publizierte Artikel aus der Feder der Arbeitsgruppe um den Pathologen James Hogg, der seit 1968 bereits mehrere Meilensteinartikel publiziert hat $[1,2]$ ?

Wir wünschen Ihnen eine anregende Lektüre.

Ihre Herausgeber der Pneumologie

\section{Literatur}

1 Hogg JC, Chu F, Utokaparch $S$ et al. The nature of small-airway obstruction in chronic obstructive pulmonary disease. N Engl J Med 2004; 350: 2645 -2653

2 Hogg JC, Macklem PT, Thurlbeck WM. Site and nature of airway obstruction in chronic obstructive lung disease. N Engl J Med 1968; 278: 1355-1360

\section{Bibliografie}

DOI http://dx.doi.org/ 10.1055/s-0030-1257059

Pneumologie 2011; 65: 723

(c) Georg Thieme Verlag KG

Stuttgart · New York

ISSN 0934-8387

\section{Korrespondenzadresse}

\section{Dr. med. Peter Kardos}

Scheffelstr. 33

60318 Frankfurt

kardos@lungenpraxis-maingau.de 